\title{
DIE BEDEUTUNG DES TODES JESU NACH DER ÜBERLIEFERUNG DES KORANS
}

Auf den ersten Blick mag es wie ein ,Datteln nach Basra tragen” erscheinen, das in jüngster Zeit häufiger verhandelte Thema ${ }^{1}$ hier um ein weiteres Mal aufzugreifen. Doch scheint mir in der bisherigen Diskussion ein Problem noch nicht genügend Aufmerksamkeit gefunden zu haben, nämlich die Frage, welchen Zweck Muhammad mit seine? Darstellung und Deutung des Todes Jesu verfolgt hat. Diesem Problern nun sind nachfolgende Betrachtungen gewidmet.

H. Räisänen hat in seinen ausgezeichneten Buch ,Das koranische Jesusbild" (Helsinki 1971) ${ }^{2}$ nachdrücklich darauf hingewiesen, daß jede Betrachtung der koranischen Überlieferung vom Tod Jesu mit dem ältesten Text, in dem dieser erwähnt wird, beginnen muß, will man möglichen Fehlschlüssen vorbeugen ${ }^{3}$. Zum ersten Mal ist vom Tod Jesu in der gewöhnlich in die zweite mekkanische Wirksamkeitsperiode des Propheten datierten Sura 19 die Rede, und zwar in v. 30/1-33/4, einem Fragment der v. 16-40/1 umfassenden Marienlegende ${ }^{4}$, in dem sich das in der Wiege liegende Jesuskind selbst vorstellt:

..(v. 30/1) Siehe, ich bin der Diener Allahs, er brachte mir das Buch und machte mich zu einem Propheten; (v. 31/2) und er machte mich zu einem Gesegneten, wo immer ich (auch) bin; und er gebot mir das Gebet und die Armensteuer (Almosen), solange ich am Leben bin, (v. 32/3) und die Liebe zu meiner Mutter; und nicht machte er mich überheblich und unglücklich ${ }^{5}$.

1 Zuletzt von K. Rudolph, Jesus nach dem Koran, in: W. Trilling/I. Berndt (ed.), Was haltet ihr von Jesus? Leipzig 1976, S. 260-287.

2 Vgl. die Rezensionen von R. Paret in: ,Zeitschrift der deutschen morgenländischen Gesellschaft" 122 (1972). S. 335 f., und K. Rudolph in: ,Theologische Literaturzeitung'" 98 (1973), Sp. $264-266$.

3 A. a. O. S. $66 \mathrm{ff}$.

4 V. $34 / 5-40 / 1$ sind medinische Hinzufügung; vgl. dazu Th. Nöldeke-Fr. Schwally, Geschichte des Qorans I, Leipzig 1909, S. 130, und R. Paret, Der Koran Kommentar und Konkordanz, Stuttgart 1971, S. $324-325$ z. St.

¿ G. Lillings, Uber den Ur-Qur'ān, Erlangen 1974, S. 225, vorgeschlagene 'Textkorrektur zugunsten einer christologischen Deutung dieser Stelle überzeugt wenig. 
(v. 33/4) Und Hil ${ }^{6}$ über mir, am Tage, an dem ich geboren wurde, am Tage, an dem ich sterben werde (amutu), und am Tage, an dem ich auferweckt werde (ub'atu) zum Leben."

Man kann diesen Text zentral nennen, denn in ihm liegt im Grunde die gesamte koranische Christologie in knapper Form zusammengefaßt vor. Doch interessiert hier vor allem zunächst nur der letzte Vers mit der Aussage vom Sterben und Auferwecktwerden Jesu. Ganz klar werden hier beide Ereignisse - das Sterben und das auferwecktwerden voneinander geschieden angekündigt. Beide freilich verbindet, daß sie genauso real sein werden, wie die Geburt es ist. Von Anfang an setzt Muhammad das wirkliche Sterben Jesu allen Doketismen zum Trotz voraus; wie auch gleichzeitig festzuhalten ist, daß Jesus, der als Gesandter Allahs für Muhammad eben nur ganz Mensch gewesen ist, sterben mußte (5, 75/9; s. dazu u.), da Unsterblichkeit nur Allach selber eigen ist ${ }^{7}$, niemals aber einem Menschen. Diese Feststellung ist insofern von Wichtigkeit, als nämlich später, und zwar in 4,157/6 - 158, etwas Gegenteilliges behauptet $\mathrm{zu}$ sein scheint:

„,(Die Juden sagten:) 'Wir haben den Christus Jesus, den Sohn der Maria Gesandten Allahs, getötet'. Aber sie haben ihn nicht getötet und nicht gekreuzigt, sondern es erschien ihnen nur so ${ }^{8}$. Und diejenigen, die darüber uneins sind, sind im Zweilfel darüber. Sie haben kein Wissen darüber, gehen vielmehr Vermutungen nach. Sie haben ihn nicht wirklich getötet. Nein, Allah hat ihn zu sich erhoben (rafa'ahu). Allah ist mächtig und weise."

Bei genauerem Hinsehen freilich ist hier kein Gegensatz zu 19, 33/4 zu entdecken; denn es wird lediglich festgestellt, wenngleich in pointierter Form: den Juden ist es dank göttlichen Eingreifens nicht gelunger, Jesus zu töten, d.h. zu kreuzigen. Daß Jesus dennoch gestorben sein kann, wird indessen mit keiner Silbe bestritten, wenn auch das rafa'ahu nicht zum Beweis dafür genommen werden kann. In ähnlichem bzw. gleichem übertragenen Sinne begegnet rafa'a nämlich sonst nur noch in $19,57 / 8$ und $3,55 / 48$. Dabei besagt das rafa'nāhu in 19,57/8 gerade nicht, daß Idris, von dem dort die Rede ist, gestorben und danach erhöht worden ist, sondern - vorausgesetzt, die Identifizierung des Idris mit dem Apostel Andreas oder dem Koch aus dem Alexanderroman trifft zu 9 - „wir haben ihn erhöht" kann hier nur bedeuten: Idris

B Zum Begriff vg1. 97, 5; 21, 69.

7 Vgl. die Belege bei J. Nosowski, Teologia Koranu, Warszawa 1970, S. 21-24.

8 Eine andere Deutung liefert Baidāwi z. St. (übers. bei H. Gätje, Koran und Koranexegese, Zürich/Stuttgart 1971, S. 174). Vgl. dazu auch R. Paret, a. a. O. S. 110 z. St. - Nur am Rande sei vermerkt, daß an diesen Satz immer wieder die Behauptung geknüpft wurde, Muhammad habe eine doketisch-gnostische Christologie vertreten. S. dazu K. Rudolph, a. a. O. S. 280.

' Vgl. J. Horovitz, Koranische Untersuchungen, Berlin/Leipzig 1926, S. 88-89; ders.. Jewish Proper Names and Derivatives in the Koran, HUCA 2 (1925), S. 175-176 . 
wurde entrückt, ohne daß er vorher gestorben ist ${ }^{10}$. Dagegen geht dem rāfi'uka in $3,55 / 48$, das wie in $4,158 / 6$ ebenfalls auf Jesus bezogen ist, die Bemerkung voraus:

„Damals ${ }^{11}$ sagte Allah: 'o Jesus! Siehe, ich lasse dich (jetzt) sterben (mutawaffika) und erhebe dich (râfi'uka) zu mir..."

Jesus ist demnach also vor seiner Erhebung gestorben. Dies bestätigt schließlich 5, 117, die jüngste Bezeugung des Todes Jesu. Hier spricht der erhöhte, vor dem Richterstuhl Allahs stehende Jesus:

"Ich redete zu ihnen (den Juden) nichts, was du (Allah) mir nicht aufgetragen hattest, sondern nur: 'Dient Allah, meinem Herrn und eurem Herrn!' Und ich war über sie Zeuge, solange ich unter ihnen weilte. Nachdem du mich hattest sterben lassen (tawaffaitani), warst du es, der über sie achthatte; den du bist über alle Dinge Zeuge."

$\mathrm{Da}$ das beidemale begegnende tawaffā im Koran allenthalben ganz eindeutig auf den Tod bezogen ist ${ }^{12}$, unterliegt es keinem Zweifel, daß es auch hier an beiden Stellen das Sterben bezeichnet. Feststeht also, Jesus ist gestorben und erst danach erhoben worden. Daß in 4, 158/6 das tawaffā verschwiegen ist, hat ganz einfach seinen Grund darin, daß der Kontext auf eine ganz andere Pointe hinauswill. In jedem Fall ist es aber legitim, in der Deutung besagten Verses auf grund von 3, 55/48 und 5,117 auch in 4, 158/6 das tawaffā dem rafa'a vorausgehen zu lassen, in dem schließlich das amütu von $19,33 / 4$ widerhallt.

Anerkennt Muhammad vorbehaltlos an allen Orten also das Faktum des Todes Jesu an, so bestreitet er doch nachdrücklich, daß Jesus eines gewaltsamen Todes gestorben, also gekreuzigt worden ist, wie in 4, 157/6 nachzulesen. Daß Jesus nach dem Zeugnis des Korans tatsächlich nicht gekreuzigt worden ist, belegen nun sicher zwei Verse; da heißt es im Vorspann zu dem bereits analysierten Vers 3, 55/48:

,Siehe, sie (die Juden) schmiedeten Ränke, aber auch Allah schmiedete Ränke, und er ist der beste Ränkeschmied." (3, 54/47)

10 Die gleiche Interpretation bietet auch die muslimische Exegese. Allerdings wird Idris hier mit Henoch gleichgesetzt, und zwar auf grund von Gen 5, 24. Doch s. auch Abu 1-Qāsim al-Husain b. Muhammad al-Isfahāni, al-mufradāt fi garibi l-Qur'ān, ed. M. S. Kilāni, Kairo 1381/1961, S. 200.

11 Der Vers, wie auch der Kontext in Sura 3, läßt sich nicht datieren. Er nimmt m. E. jedoch mit an Sicherheit grenzender Wahrscheinlichkeit auf. 4, 157/6-158 Bezug, ist also jünger, wie in 3, 54/47 zweifellos gleichfalls auf die versuchte Kreuzigung angespielt ist. Vgl. auch 5, 110 (s. u.).

12 tawaffä ist zunächst term. techn. für das ,Wegnehmen der Seelen" durch die Todesengel $(4,97 / 9 ; 6,61 ; 8,50 / 2=47,27 / 9 ; 16,28 / 30.32 / 4 ; 32,11 ; 71,37 / 5)$, beschreibt dann aber auch das Handeln Allahs $(10,46 / 7=13,40=40,77 ; 12,101 / 2$; $10,104 ; 16,70 / 2=22,5 \mathrm{~b}=40,67 / 9 ; 39,42 / 3 \mathrm{a})$ und bezeichnet schließlich das Sterben selbst $(2,234.240 / 1 ; 3,193 / 1=7,126 / 3)$. Vgl. auch den Parallelismus gä'a l-mautu $(6,61)$ // tawaffă l-mautu $(4,15 / 19)$. 
Worauf sollte sich hier das Ränkeschmieden (der Juden) beziehen, wenn nicht auf die (versuchte) Kreuzigung? Die Richtigkeit dieser Annahme bestätigt 5, 110, gleichsam Muhammads Interpretation zu 3, 54/47. Dort spricht Allah zu dem erhöhten, vor seinem Richterstuhl stehenden Jesus U.a.:

„Damals ${ }^{13}$, als ich die Kinder Israel von dir zurückhielt (kafaftu), als du ihnen die klaren Beweise gebracht hast..."

Da kaffa im Koran nur entweder Allahs Verhinderung einer gewaltsamen Behandlung von Menschen $(4,84 / 6 ; 5,11 / 14 ; 48,20.24)$ oder aber das Sich-enthalten der Menschen von eben solcher Behandlung (4, 77/9. 91/3) bezeichnet ${ }^{14}$, kann in unserem Vers folglich nur gemeint sein: den Juden ist es infolge einer Intervention Allahs nicht gelungen, Jesus gewaltsam zu Tode zu bringen, d.h. ihn zu kreuzigen.

Als gesichert darf daher gelten: Jesus ist nach koranischer Überlieferung wirklich gestorben, aber er wurde nicht gekreuzigt ${ }^{15}$. Wie $\mathrm{Mu}$ hammad sich dann aber die in 4, 157/6 erwähnte Kreuzigungsgeschichte genau vorgestellt hat, können wir nicht wissen, da hierüber keinerlei Notiz im Koran $\mathrm{zu}$ finden ist. Muhammad unterstellt den Juden nur, sie hätten Jesus gekreuzigt; in Wirklichkeit freilich haben sie nur einen Doppelgänger - nach Johannes Damscenus einen „Schatten” (De haeres. 100) ${ }^{16}$ hingerichtet ${ }^{17}$. Sie konnten Jesus nicht kreuzigen, weil Allah ihnen zuvorgekommen ist. Er errettete Jesus und erhob ihn zu sich. „Will man sich das konkret vorstellen" - schreibt H. Räisänen, a.a.O.S. 73 - „ist die Annahme am wahrscheinlichsten, Gott habe Jesus nach seiner Errettung (eines natürlichen Todes) sterben lassen und ihn dabei zu sich genommen".

Trifft die bisherige Analyse der den Tod Jesu berichtenden Texte soweit zu, gilt es nun zu klären, in welchem zeitlichen Zusammenhang Tod und Auerweckung (19, 33/4) bzw. Erhebung (3, 55/48; 4, 158/6).

Der älteste Beleg - 19, 33/4 - unterscheidet deutlich zwischen dem Tag des Sterbens (yaum amūtu) und dem Tag des Auferwecktwerdens (yaum ub'atu); beide Ereignisse sind hier zeitlich klar voneinander getrennt. Darüberhinaus legt die Tatsache, daß an dieser Stelle das Verb ba'ata für die Auferweckung Jesu gebraucht ist, nahe, daß Muhammad die Auferweckung Jesu offenbar als ein Ereignis der allgemeinen Totenauferweckung am yaumu l-ba'ti $(30,56)$ verstanden wissen will, wie ja $b a^{\prime} a t a$ einer der termini technici für die Auferweckung der Toten durch

\footnotetext{
13 S. o. Anm. 11.

14 Vgl. al-Isfahāni, a. a. O. S. $433 a$.

15 Vgl. H. Räisänen, a. a. O. S. 71; K. Rudolph, a. a. O. S. 280-281.

16 PG 94, 765b; Zitiert bei K. Rudolph, a. a. O. S. 280 Anm. 44.

17 So Baidāwi, a. a. O. (s. o. Anm. 5). Vgl. auch J. Nosowski, a. a. O. S. 153-154.
} 
Allah am Jüngsten Tage ist ${ }^{18}$. Dagegen darf man nach 3, 55/48, erst recht nach 4, 158/6, annehmen, Tod und Erhebung Jesu ereigneten sich in unmittelbarer Folge; ein zeitlicher Abstand zwischen beiden wird jedenfalls nicht erwähnt. Der Tag des Todes und der Tag der Erhebung sind hier - wie es scheint - identisch miteinander. Ist diese Beobachtung richtig, können wir hinsichtlich des Zeitpunktes der Auferweckung/ Erhebung Jesu in den jüngeren Belegen immerhin ein Abrücken Muhammads von der älteren Anschauung konstatieren, das bemerkenswert ist. Unterstrichen wird dieses Abrücken nicht zuletzt durch das nunmehr gebrauchte rafa'a anstelle von ba'ata, auf dessen Bedeutung oben bereits hingewiesen worden ist. Fragt man nun, was diese Wandlung in der Anschauung Muhammads bewirkt haben wird, so ist es sicher die Bedeutung gewesen, die er der Geschichte vom Sterben und Auferwecktwerden/Erhobenwerden Jesu beigemessen hat. Zwar mag man auch nur, wie K. Rudolph angedeutet hat, dahinter einen ,schwache (n) Nachhall der christlichen Auferstehungs- und Himmelfahrtslehre" vermuten wollen/können ${ }^{19}$, aber damit ist doch nicht alles gesagt; denn der skizzierte - scheinbare - Widerspruch hinsichtlich des Zeitpunktes der Auferweckung/Erhebung zwischen 19,33/4 und 3, 55/48; 4, 158/6 klärt sich sehr bald auf, wenn man die Abzweckung der jeweiligen Kontexte berücksichtigt. Und da wird deutlich, daß Muhammad wenigstens dreierlei mit seiner Darstellung und Deutung des Todes Jesu intendiert hat.

Beginnen wird wiederum mit dem ältesten Beleg $(19,33 / 4)$, dem Vers, der den Abschluß der Selbstvorstellung des in der Wiege liegenden Jesuskindes bildet $(19,30 / 1-33 / 4)$. Eine gewisse antichristliche Tendenz wird man dieser Perikope schwerlich absprechen können oder wollen, insofern nämlich, als hier auf Jesu ausschließliches Menschsein (vgl. 43, 59 20; 9, 30-31) rekurriert wird. Wenngleich nach Muchammads Anschauung die Geschöpflichkeit Jesu, sein Menschsein, ihren Ursprung im unmittelbaren Wirken Allahs hat $\left(3,59 / 2{ }^{21} ; 19,17 ; 21,91 ; 66,12 ; 4,171 / 69 ; 3\right.$, 45/0), bleibt trotzdem seine Grundüberzeugung: „Gott ist nicht der Vater, sondern der Schöpfer Jesu" 22. Daher kann Muhammad Jesus denn auch mit den Tugenden charakterisieren, die einen Muslim auszeichnen (19, $30 / 1-31 / 2$; vgl. 5, 111). Zudem zeigt, daß Gleiches u. a. auch von Johannes dem Täufer mitgeteilt wird $(19,12 / 3-15)$, daß Jesus ganz in die

18 Die Belege sind Muhammad Fu'ād 'Abdalbāqi, al-mu'gam al-mufahras li-alfāz al-Qur'ān al-karim, Kairo 1364 H., S. 124b-125a, zu entnehmen.

19 A. a. O. S. 281.

20 Der Text ist etwa gleichzeitig mit 19, 30/1-33/4. Vgl. dazu die medinische Ergänzung 19, 34/5-36/7 sowie die Belege bei K. Rudolph, a. a. O. S. 270. 282-283.

21 Vgl. dazu die zutreffende Bemerkung J. Nosowskis, a. a. O. S. 153, und R. Paret, a. a. O. S. 70 z. St.

22 H. Räisänen, a. a. O. S. 77. 
Reihe der Propheten aufgenommen ist, plaziert zwischen Johannes dem Täufer und Muhammad (vgl. 61, 6; 42, 13/11; 6, 85). Weil Jesus für Muhammad wie alle Propheten eben nur Niensch war, deshalb stirbt er auch wie ein Mensch:

„Christus, der Sohn der Maria, ist nur ein Gesandter, und schon vor ihm sind Gesandte dahingegangen." (5, 75/9)

Schließlich wird er am „Tage der Auferweckung” $(30,56)$ wie alle Menschen auferweckt werden zum Leben. Von hier aus wird verständlich, warum in 1933/4 nicht von der „Erhöhung” Jesu gesprochen wird, sondern nur davon, daß „er zum Leben erweckt wird”. Als ein Mensch teilt er das Schicksal aller Menschen und wird wie sie alle auch auferweckt werden, um vor dem Richterstuhl Allahs zu erscheinen $(5,110-$ 117). Von einer „Heilsbedeutung” des Todes Jesu weiß Muhammad nichts ${ }^{23}$. Der auferstandene, richtiger natürlich: der auferweckte Jesus hat lediglich die Aufgabe, die auch die anderen Propheten zu erfüllen haben, nämlich als Ankläger gegen die „Ungläubigen” im Gericht aufzutreten $(4,159 / 7)^{24}$. So dient die Darstellung vom Tode Jesu zunächst einmal dazu, sein ausschließliches Menschesein zu demonstrieren.

Abrenzung gegen jede Form christlicher Interpretation des Todes Jesu als Heilsereignis, ja die Ablehnung derselben a priori ist freilich erst ein Aspekt in Muhammads Darstellung. Betrachten wir nun vor allem 3, 54/47-55/48 und 4, 157/6-158 (159/7), einschließlich des Nachhalls in 5, 110, fällt auf, daß diese Perikopen - zumindest vordergründig - von einer antijüdischen Grundhaltung geprägt sind: Den Juden ist es nicht gelungen, Jesus zu töten, zu kreuzigen - klingt unüberhörbar deutlich aus diesen Sätzen heraus. Ganz abgesehen davon, daß Muhammads hier ausgesprochene, radikale Bestreitung der Kreuzigung um ein weiteres Mal gegen eine christliche teologia crucis argumentiert, würde man die antijüdische Ausrichtung genannter Perikopen allerdings völlig mißverstehen, wollte man sie deswegen als Sympathiebekundung oder Apologie für Jesus deuten. Davon kann keine Rede sein; denn das hier zugrunde liegende "Leitmotiv" ist nämlich einziger jener — oben schon herausgestellte — Satz, daß Allah es gewesen ist, er allein und niemand anders, durch dessen Eingreifen die Pläne der Juden zunichte gemacht worden sind $(3,54 / 47)$, und zwar nicht aus Gründen besonderer

2 Vgl. W. M. Watt, The Christianity criticized in the Qur'än, "Muslim World" 57 (1967), S. 197-201.

$\because 443,61$ gehört $m$. E. nicht in einen Zusammenhang mit 4, 159/7. Wenn sich dort das Suffix in innahu auf Jesus bezieht, dann nur in der Weise daß Jesus durch seine Botschaft dem ,Wissen von der Stunde" dient. Anders freilich Baidāwi z. St. (übers. bei H. Gätje, a. a. O. S. 175-176. - Vgl. auch K. Rudolph, a. a. O. S 269, 282, und die dort angegebene Literatur. 
Bevorzugung oder Wertschätzung Jesu - auch wenn es gelegentlich im Koran heißt, er sei ein auf Erden Angesehener (wagih) und ein im Jenseits Allah Nahestehender (mina 1-muqurrabina; 3, 45/0) ${ }^{25}$, sondern zunächst einfach deshalb, weil Jesus einer der Gesandten Allahs ist, die von ihm mit besonderem Schutz umgeben sind (vgl. 3, 146/0-148/1). Ferner aber, und das ist das letztlich Entscheidende, bestimmt einzig und allein Allah über Leben und Tod der Menschen, einschließlich seiner Gesandten. Nur diejenigen sterben oder nur deren Tod läßt er geschehen, für die er ihn vorherbestimmt hat $(3,145 / 39 ; 57,22)$. So gesehen, stellen jene o. e. Perikopen aus der Geschichte vom Sterben und Erhobenwerden Jesu keine antijüdische Propaganda dar, sondern sind in Muhammads Predigt ein eindrucksvoller Beweis für die Allmacht Allahs, verkündigt ad maiorem Dei gloriam!

Darüberhinaus aber hat der Tod Jesu nach der koranischen Überlieferung - wie mir scheint - noch eine dritte, und zwar auf Muhammad selber zielende Bedeutung. Dabei handelt es sich — das sei vorweg betont - nicht um irgendeine Form von Typologie, etwa um den Versuch, hier in Jesus einen „typos tou mellontos” (Röm 5, 14) aufzuweisen. Der bloße Gedanke schon an eine solche praefiguratio ist Muhammad absolut fremd. Dessenungeachtet dienen die Hinweise auf die Schicksale der Vorgänger Muhammads und die unbestreitbaren Vergleiche dieser mit seinem eigenen gelegentlich wohl dazu, eo ipso sein eigenes verstehbar werden zu lassen; doch an keiner Stelle wird damit zugleich der Versuch einer typologischen Auswertung bzw. Ausnutzung unternommen ${ }^{26}$. Trotzdem aber hat der Tod Jesu für Muhammad persönliche Relevanz.

Der Gedanke an den eigenen Tod ist für Muhammad offensichtlich ein Problem gewesen, das ihn des öfteren ernsthaft beschäftigt hat 27 . Daher überrascht es nicht, daß er einmal sogar seine Getreuen fragen konnte:

„Was denkt ihr: Ob Allah mich und die mit mir sind, vernichtet, oder wird er uns schonen? Wer wird dann die Ungläubigen von der schmerzlichen Strafe retten?" $(67,28)$

$2 \pi$ muqarrabuna heißen aber auch einfach alle diejenigen, die ins Paradies eingehen werden (z. B. $56,11.88 / 7 ; 83,28)$, ohne daß sie dadurch besonders qualifiziert währen. Daßs muqarrabuna freilich noch andere Bedeutungen hat, hat $u$. a. J. Horovitz, Das koranische Paradies, Jerusalem 1923 (= SH I, 6), S. 2, vermerkt. Zur Position Jesu vgl. auch 3,59/2 und dazu o. Anm. 21.

$\because$ G. E. von Grunebaum, Die Erfahrung des Heitigen und die Auffassung vom Menschen, in: Studien zum Kulturbild und Selbstverständnis des Islams, Zürich/ /Stuttgart 1969, S. 310 Anm. 3.

${ }_{27}$ Vgl. das Zitat aus Ibn Hisām bei T. Andrae, Mohammed, sein Leben und sein Glaube, Göttingen 1932, S. 139. 
Zwar ist der Text schwer (gar nicht?) zu datieren ${ }^{28}$, der Kontext aber weist deutlich auf einen eschatologischen Zusammenhang hin ${ }^{29}$, von dem her wohl auch der Vers gedeutet werden will. Zwei Fragen stehen vor diesem eschatologischen Hintergrund: Sind Muhammad und die Seinen unsterblich? Kann ein gestorbener Gesandter Allahs noch Fürsprecher im Gericht sein? Die zweite Frage hier beantworten zu wollen, würde weit über den gesetzten Rahmen hinausgehen ${ }^{30}$; zudem gehört sie schwerlich in unseren Zusammenhang. Eine wenigstens teilweise Antwort erhält sie aber von der Antwort auf die erste Frage her, und darauf muß sich Muhammad antworten lassen:

„Nicht haben wir für einen Menschen vor dir (Muhammad) Unsterblichkeit bestimmt. Wen du also stirbst, sollen dann die Ungläubigen unsterblich sein? Nein, jede Seele wird den Tod schmecken!" (21, 34/5--35/6)

„Siehe, du (Muhammad) bist ein Sterblicher, und diese (die Ungläubigen), siehe, sie sind Sterbliche." $(39,30 / 1)$

Während in der Frage von 67, 28 Sterblichkeit und Tod Muhammads nicht mehr als eine abstrakte Möglichkeit zu sein scheinen, wird in den darauf gegebenen Antworten unter Verweis auf die Sterblichkeit aller Menschen $(21,35 / 6=29,57=3,185 / 2$ Anfang) ganz klar festgestellt, daß Muhammad - wenngleich er auch Gesandter Allahs ist - als Mensch eben keine Ausnahme darstellt, Gläubige wie Ungläubige gleichermaßen dem Gesetz der Sterblichkeit aller unterliegen.

Besondere Beachtung verdient hier ein Vers, in dem die Motivation und gleichzeitig auch die Konsequenzen daraus vorgetragen werden:

,Muhammad ist nur ein Gesandter, und schon vor ihm sind Gesandte dahingegangen ${ }^{31}$. Wenn er also stirbt oder ermordet wird, werdet ihr euch dann von ihm abwenden?" (3, 144/38) ${ }^{32}$

Zunächst gilt in Erinnerung zu rufen, daß der erste Satz wortwörtlich in 5, 75/9 auf Jesus bezogen ist, sein Sterben also genauso begründet wird wie das Muhammads. Diese Gleichartigkeit der Motivation ist gewiß nicht zufällig und besitzt nach beiden Richtungen hin Relevanz: Das, was über den Tod Jesu mitgeteilt ist, gilt in gleicher Weise auch Muhammad, wie umgekehrt auch der Bericht über das mögliche Ende Muhammads und dessen Begründung für die Interpretation des Todes Jesu

25 Th. Nöldeke - Fr. Schwally, a. a. O. S. 133.

24 R. Paret, a. a. O. S. 484 zu 67, 27.

30 Vgl. dazu L. Gardet, Islam, Köln 1968, S. 84-86.

3 Zum Hintergrund dieses Satzes vgl. Th. Nöldeke-Fr. Schwally, Gescirichte cies Qorans II, Leipzig 1919, S. 82, sowie W. M. Watt, Bell's Introduction to the Qur'ān, Edinburgh 1970 (= Islamic Surveys 8), S. 51-52.

:" Man fühlt sich hier an die Frage Jesu aus Joh 6, 67: ,Wollt ihr auch weggehen?", erinnert. 
durch Muhammad Bedeutung hat. Wie Jesus nach dem Beschluß Allahs gestorben ist, so wird auch Muhammad sterben, wie Allah es bestimmt. Die Frage ist nur: Was wird aus den Gläubigen? Werden sie sich nach seinem Tod von ihrem Glauben abkehren? ${ }^{33}$ Zweifellos sah Muhammad in seinem möglichen Tod - der Kontext des Verses läßt in seiner Bezugnahme auf die für die Muslime nicht glücklich ausgegangene Schlacht am Uhud-Berg immerhin damit rechnen ${ }^{34}$ - eine echte Gefahr, nämlich einen drohenden Auseinanderfall der erst jungen muslimischen Gemeinschaft. Daß Muhammads Sorge keinesweg unbegründet sein sollte, sollte der Gang der Geschichte bestätigen; denn nach dem Tode Muhammads brach der Konflikt unter den Muslimen offen aus: die ridda, die sog. Abfallbewegung vom Islam zeigte nämlich in aller Deutlichkeit, daß die ersten Muslime sich zunächst mehr aus einem Gefühl der Solidarität heraus der Person des Propheten als dem neuen Glauben verpflichtet fühlten ${ }^{35}$, Insofern mußte der Tod Muhammads folgenschwere Bedeutung für den Bestand der islamischen Gemeinschaft haben. Nun wird man dieses vaticinium ex eventu nicht in den Korantext hineinlesen dürfen, doch vermag es das Problem zu veranschaulichen, um das es hier geht. Demonstriert es doch ein fundamentales Mißverständnis der ersten Getreuen Muhammads, in dem sie den neuen Glauben mit seiner Person verknüpften, anstatt ihn ausschließlich als „Hingabe an Allah” zu verstehen ${ }^{36}$, wie es Muhammad, ohne müde zu werden, verkündete. Und hier trifft er sich seiner Meinung nach mit Jesus, der nach koranischer Uberlieferung gesagt hat:

„Ungläubig sind die, die sagen: 'Allah, das ist der Christus, der Sohn der Maria'. Christus aber hat gesagt: 'Ihr Kinder Israel! Dient Allah, meinem Herrn und eurem Herrn...' " $(5,72 / 6=5,117) .{ }^{37}$

Nur die ,Sekten” (aḩzāb) haben die Wahrheit dieses Satzes bestritten und Christus zum Gott gemacht (43, 65; vgl. 3, 64/57) ${ }^{38}$. Für Muhammad liegt in dieser Feststellung eine Bestätigung seiner eigenen Mission, insofern nämlich, als er Jesus vom ,Vorwurf” der Selbstvergottung ,,befreit" hat und damit zugleich seine eigene Verkündigung als die echte jesuanische Nachtfolge deklarieren konnte. Unter diesem Gesichtspunkt

33 Eine andere Deutung notiert R. Paret, a. a. O. S. 31 z. St.

34 W. M. Watt, a. a. O. S. 52.

3. G. E. von Grunebaum, Der Islam in seiner klassischen Epoche, Zürich/Stuttgart 1966, S. $63 \mathrm{ff}$.

36 D. Z. H. Baneth, What did Muhammad mean when he called his Religion "Islam"? IOS 1 (1971), S. 183-190.

37 Vgl. $3,51 / 44 ; 9,30-31 ; 43,64$.

3s Wie der Satz 5, 111 verstanden werden muß: "Glaubt an mich (Allah) und meinen Gesandten (Jesus)", lehrt 3, 50/44: Fürchtet Allah und gehorcht mir (Jesus)". Es geht also nicht um einen Glauben an Jesus im christlichen Verständnis. 
gewinnt schließlich auch der Tod Jesu (besonders für Muhammad) paradigmatische Bedeutung, und zwar als das natürliche Lebensende eines treuen Gesandten Allahs, der wie er unermüdlich zum Glauben an den einen Gott gerufen hat. Hier weiß sich Muhammad bis zum Tod als Nachfolger Jesu und schließt so die lange Reihe der Gesandten Allahs ab: Als „der erste der Muslime” (6, 162/3-163) ist er zugleich ,das Siegel der Propheten" $(33,40)$. So gesehen, läßt der Tod Jesu Muhammads eigenes Ende erkennen und begreifen ${ }^{39}$ (vgl. 3, 146/0-148/1) *.

39 Vgl. T. Andrae, a. a. O. S. 146.

* Manuskript abgeschlossen am 10. 12. 1976 